\title{
Design of a miniature Servo Controller for a Flow Control Actuator in Aerospace Application
}

\author{
${ }^{1}$ Zaki Anwar, ${ }^{2}$ Y. Laxminarayana, ${ }^{3}$ J.V.Sudheendra, ${ }^{4}$ R.V.Naveen Kumar \\ $1,2,3,4$ Scientist -RCI/DRDO, Hyderabad \\ Email: ${ }^{1}$ Zaki.Anwar@rcilab.in, ${ }^{2}$ LaxmiNarayaana.Y@rcilab.in
}

Received: 10 ${ }^{\text {th }}$ Feb 2018, Accepted: $20^{\text {th }}$ March 2018, Published: 30 $^{\text {th }}$ April 2018

\begin{abstract}
This paper deals with the design of a miniature servo controller for a short duty flow control actuator. The servo controller is designed for position control of the flow control actuator (FCA) with high accuracy. The flow control actuator in this application demands quick response, fine precision and compact size. The controller is required to be confined and integrated inside the FCA casing wherein space is shared along with the BLDC motor, gears assembly and position sensor. The servo controller is realized and the Tests results are meeting the specifications.
\end{abstract}

\section{Keywords}

Flow Control Actuator, BLDC motor, Servo Controller

\section{Introduction}

An actuator, in general, is a device that produces some sort of physical motion by taking energy from a source. The actuators are characterized based on their sources of energy. Hydraulic, pneumatic or electromechanical actuators are more common among others which use liquid, gas or electrical power as their energy source respectively whereas the type of motion these actuators produce can be linear, rotary or any other combination of these motions. In some aerospace vehicle applications, actuators are employed for precise control of opening of vanes for flow of gases thereby controlling the thrust as per requirement of the vehicle. The aerospace vehicle under consideration is thrust controlled by Vane of a flow control mechanism operated by an actuator. The functional block diagram of this flow control actuator (FCA) system is as shown in Fig-1.The FCA is realized as the electromechanical actuation system, owing to very narrow space constraints as shown in Fig2,.In this FCA system, the BLDC motor acts as prime mover and uses electrical energy supplied by battery to drive the vane through gear trains. Gear trains serve speed reduction and the amplification in the torque produced by the motor.
The servo controller is very essential and key element of any actuation system as it controls the position of the output depending upon the input command which in turn controls the entire system. Therefore, it is ultimately in charge of entire aerospace vehicle as it closes the inner loop of control system. The servo controller sends required voltage signals to drive circuitry of the motor which are proportionate to the error between the command and the actual position of the vane sensed by the position sensor. The critical requirement of the controller is to have fast response characteristics for position command along with providing the stability.

As the brushless (BLDC) dc motor lacks the brushes for commutation, it is the job of controller to execute this commutation function, so it energizes the proper phases of the motor in a sequence calculated by Hall sensors as the rotor moves. The controller implements a PWM (pulse width modulation) scheme to power the motor. The controller eventually performs the closed loop function to execute position control to suit the requirement of FCA system which demands high bandwidth and fast dynamic response.[4][6]

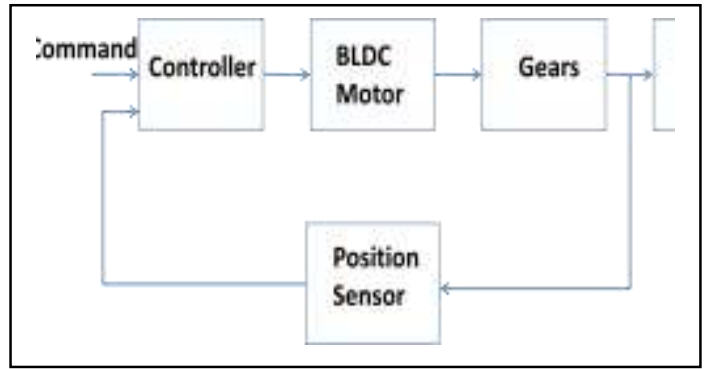

Fig-1. Functional block diagram of FCA

In this given aerospace application, the space for FCA itself is very limited; hence the space for controller to be designed is further acute and narrow. The specifications of BLDC motor to be controlled are given below in table-1. [1] 


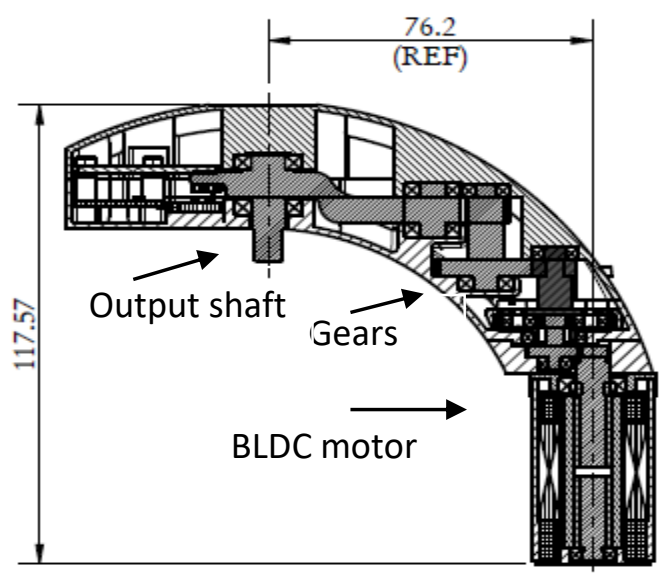

Fig-2. Space envelope for FCA actuation system (all dimensions are in $\mathrm{mm}$ )

Table-1 Specifications of Brushless DC Motor:

\begin{tabular}{|l|l|}
\hline \multicolumn{1}{|c|}{ PARAMETER } & \multicolumn{1}{|c|}{ SPECIFICATIONS } \\
\hline Voltage & $28 \mathrm{~V} \mathrm{DC}$ nominal \\
\hline Kt & $0.05 \mathrm{Nm} / \mathrm{A}$ \\
\hline Peak Current & $5 \mathrm{~A}$ \\
\hline No Load Speed & $5000 \mathrm{rpm}$ \\
\hline Full Load Speed & $>3300 \mathrm{rpm}$ at $0.1 \mathrm{Nm}$ \\
\hline Gear ratio & $200: 1$ \\
\hline Position Sensor & Rotary potentiometer \\
\hline
\end{tabular}

Servo Controller Design

The servo controller has to drive the FCA actuation system very accurately in order to control the position of vane after receiving the command from the on-board computer (OBC) of the vehicle. The control logic is implemented in the servo controller to drive the motor as per command to attain the desired position.

There are op-amp circuits at the input to compute the error by addition or subtraction of input position command from the $\mathrm{OBC}$ and position feedback from potentiometer sensor closing the loop. The error thus calculated is processed by further circuitry until it becomes minimal. The servo controller subsequently makes required operation to minimize the error based on some control logic generally proportional, integral or derivative or a combination of these three. The factors used in this logic must be adjusted according to the requirement of the system. The servo controller takes control actions specifically for the error processing depending upon the tuning of logic parameters. The controller response is described in terms of the sensitivity of the controller to an error, the extent to which the controller overshoots the set point and the degree of system oscillation.
The servo controller switches winding current in BLDC motors based on status of three Hall sensors which update rotor position. This is called electronic commutation. The rotor in a usual BLDC motor consists of permanent magnets while the stator is made up of three-phase windings and three Hall-effect sensors which depending upon the pole passing by, turn on and off indicating the position of the shaft. With this information, the controller using semiconductor switches, is able to switch the current in each winding at the required timings. [1][3][4]

The design involves from circuit simulation to select proper control logic and parameters gains, selection of suitable electronic components with respect to voltage, current and power ratings, designing suitable electronic protection circuits. Apart from it working temperature ranges of components and heat sink also require consideration. Also simulation of complete actuation system is to be done to check the drive under load and no load conditions to match and tune the controller.

Hardware Realization

The controller hardware is designed into control and power section. The control section incorporates control logic and consists of error amplifier, compensator and PWM generator while power section incorporates commutation logic and employs semiconductor power switches in hex-bridge, gate drivers, converters and protection circuits. Electronic components are selected based on their input, output voltage, current or power characteristics and operating temperature ranges. Besides these, some issues regarding compactness of controller have also been considered. [2][3]

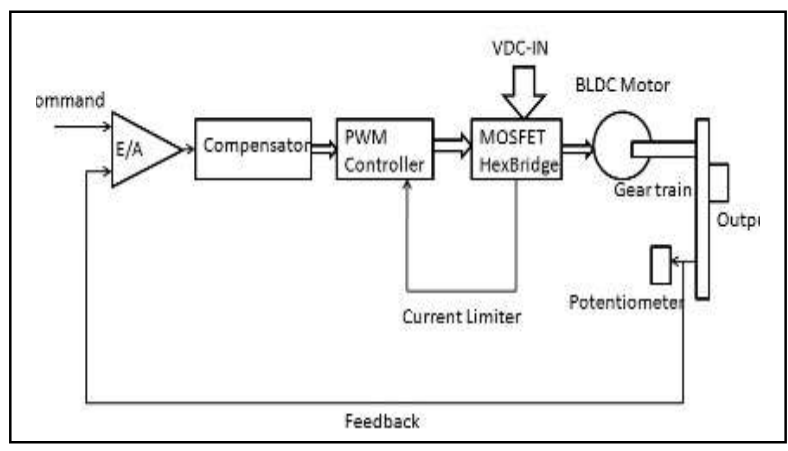

Fig-3. General structure of servo controller for actuator

This servo controller has been centrally designed around L6235 IC in which control logic, MOSFET hex-bridge and gate drive circuitry are embedded into single chip that simplify and optimize motor control. For driving three-phase brushless dc motors, the L6235 integrates a threephase bridge, high speed PWM current controller 
and decoding logic for 120 degree Hall sensors. These features allow to drastically reduce the external components count to meet space constraints. The chip also features non dissipative over current protection on the high-side power MOSFETs and thermal shutdown.[5][6][7][8]

Remaining error amplifier circuit has been realized using MC33079D, a quad Op-Amp IC, saving space. Wide range DC-DC converter ITW2415S is used in power conversion section.[6]

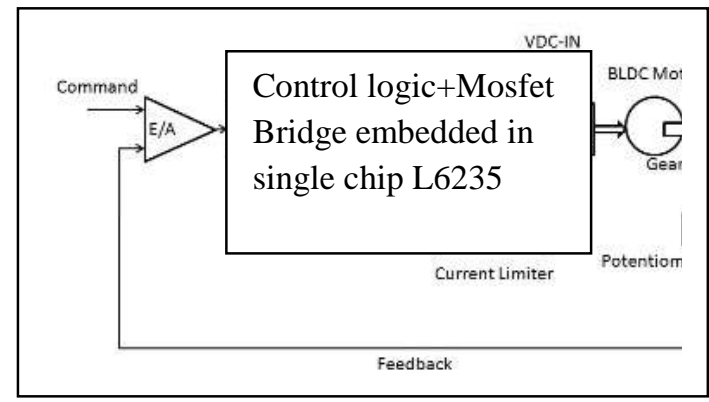

Fig-4. simplified structure of implemented controller

Hall effect sensors, 120 degree electrically apart, are open collector devices so they require a pull up resistor from the collector to a positive supply voltage. In this case the sensor is pulled up to +5 volts using $10 \mathrm{k} \Omega$ pull up resistors. [6]

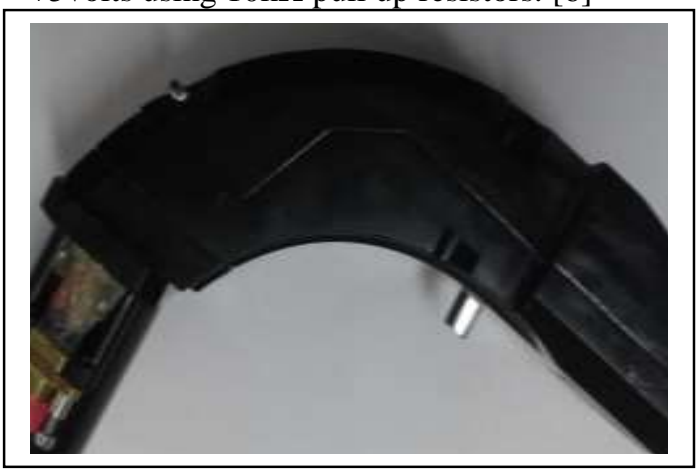

Fig-5. FCA closed view

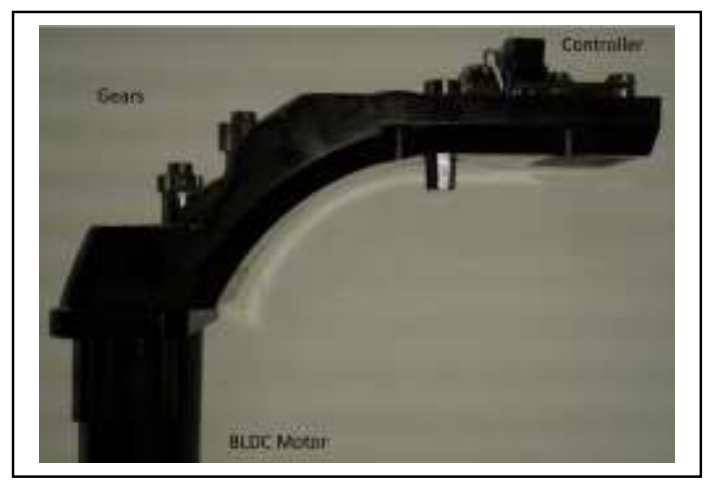

Fig-6. FCA opened - side view

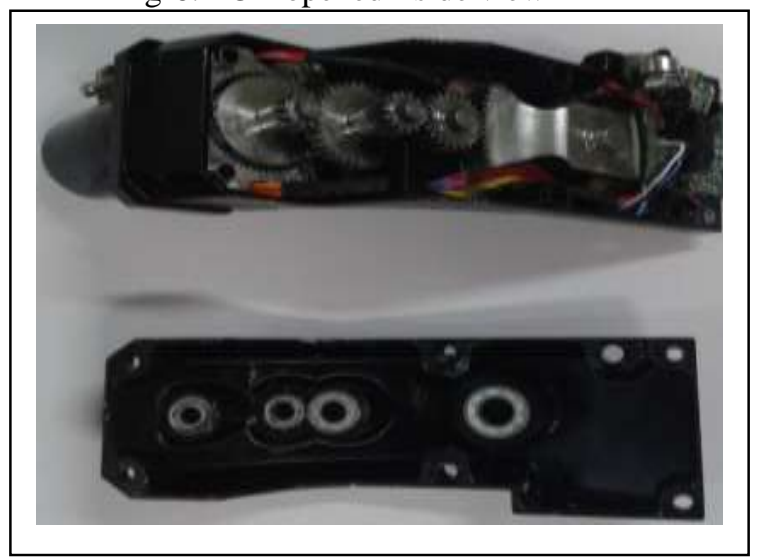

Fig-7. Controller arrangement in actuator

Apart from the above mentioned considerations some other factors that were considered to achieve compactness were going for multilayer PCBs, avoiding unnecessary circuitry wherever possible since acute shortage of space. The actuator body itself has been used as heat sink for the electronic circuitry. Moreover, PWM switching frequency has been kept high to filter out minute ripples thereby reducing the size of line filters. [6]

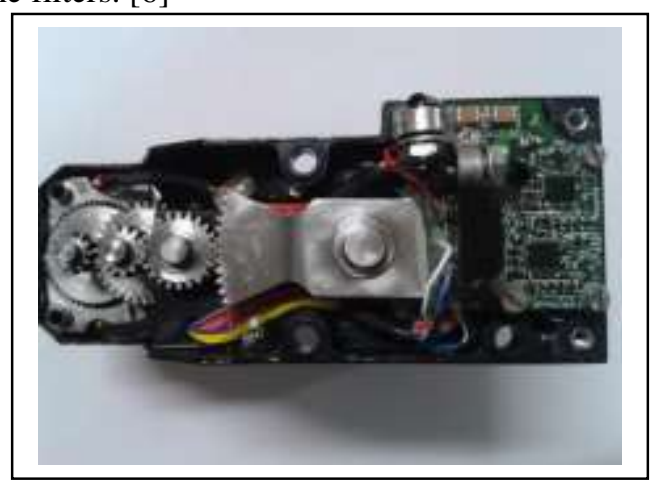

Fig-8. Controller PCB fitted in actuator housing

\section{Hardware Results Of Controller Implementation}

To evaluate the performance of the actuator integrated with controller, hardware functional tests are performed and results are shown in below figures-8 and 9. The closed loop controller for Actuator is modelled using MATLAB/Simulink. [2] 


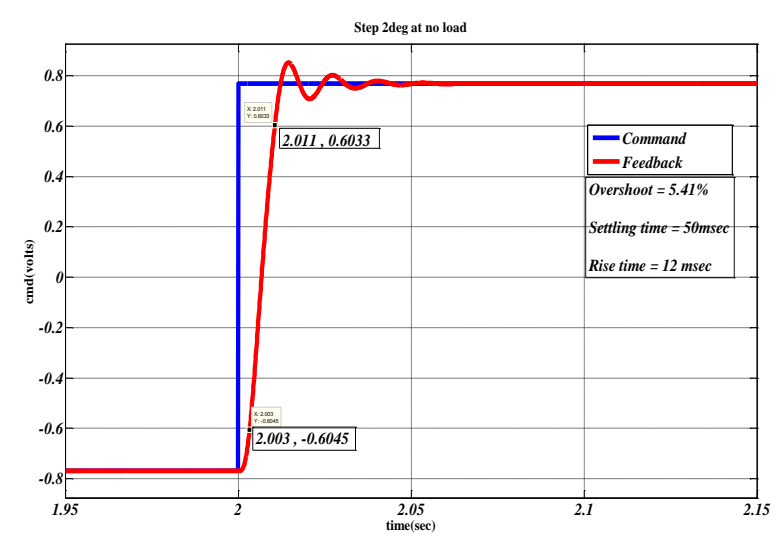

Fig-9. Step response at 2degree

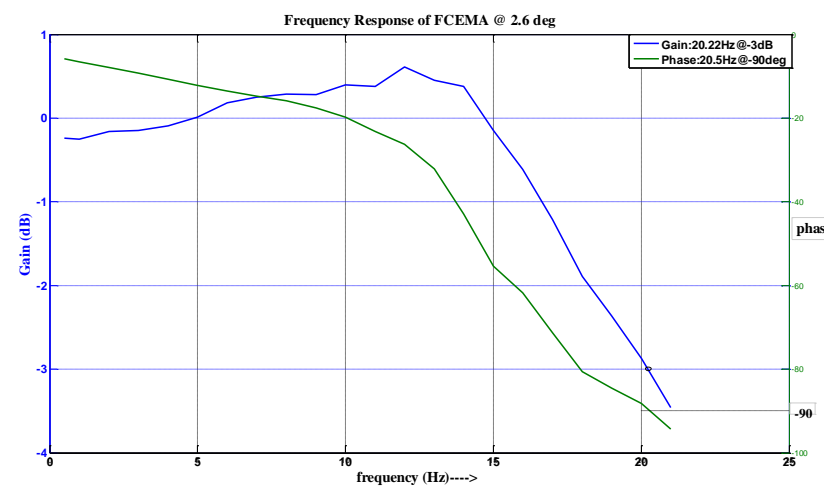

Fig-10. Frequency response at 2.6 degree

\section{Conclusion}

In this paper, design of a compact servo controller using L6235 for rotary actuator control is implemented. The controller IC, L6235 provides high speed PWM. It is a good solution for motor control where acute space constraints exist. The selection of PWM frequency and control logic parameters i.e. gains are critical in designing a servo controller, therefore they need be optimized through experiment.

The servo controller for actuator is designed within the compact size, hardware is realized and the system is integrated and tested. The method proposed in this paper is simple and reliable. The hardware test results at no-load and load conditions under different conditions are satisfactory and hence the controller is able to meet the requirements of the servo system for aerospace application.

\section{References}

[1] Design of BLDC Motor by Hensalman.

[2] Modern Control Systems by K.OGATA

[3] A BLDC motor controller with current-loop control by Siyuan Gao

[4] Vandana Govindan T.K, Anish Gopinath and S.Thomas George, 'A Simple commutation method for PMBLDC motor used in Speed
Servo System', 978-1-4244-93944/11/\$26.00@2011 IEEE,pp.340-345.

[5] Smart Drivers Simplify and Optimize Motor Control by By David Morrison, Editor, Power Electronics Technology

[6] Design of a Compact Servo Controller for a short duty Aerospace Application by Zaki Anwar, Y.Laxminarayana, J.V.Sudheendra \& R.V.Naveen Kumar.

[7] L6235 Three Phase Brushless Dc Motor Driver by Vincenzo Marano

[8] L6235 Datasheet and Application 